\title{
Dynamic response of a detonation vessel
}

\author{
B. Simoens ${ }^{1}$, M. H. Lefebvre ${ }^{1} \&$ F. Minami ${ }^{2}$ \\ ${ }^{1}$ Laboratory for Energetic Materials \\ Royal Military Academy, Brussels, Belgium \\ ${ }^{2}$ Graduate School of Engineering \\ Materials and Manufacturing Science \\ Osaka University, Osaka, Japan
}

\begin{abstract}
Detonation vessels are used for a wide range of applications. They are generally designed for a specific use and are built to withstand the impulse induced by the detonation of a given maximum mass of explosive. In general however, the characteristics of the explosive charge however are not specified. It is assumed to be spherical and is usually expressed as a TNT-equivalent mass of explosive. Extensive testing of various explosive charges in a small scaled cylindrical vessel has led to several important recommendations for a better (more rational) use of impulsively loaded vessels. The shape of the explosive charge has been shown to be crucial in the loading on the vessel wall: peak deformations can vary by a factor of two with varying charge shape. A "worst case" charge shape can be found for every vessel. Experiments have shown as well the large benefit of splitting one large charge into two half charges separated by a certain distance: the vessel response is significantly reduced in the latter case. Changing the charge location in a cylindrical vessel for different shots also allows to extend the vessel lifetime by spreading the location of largest deformation over a large area of the vessel wall instead of concentrating the impulse on the same location for every shot. Finally no major influence of the location of the initiation of the charge has been observed. Keywords: detonation vessel, impulsive loading, vessel response, cylindrical charge, explosive emulsion, initiation location, split charges.
\end{abstract}




\section{Introduction}

Pressure vessels are widely used worldwide. The vessel response to static pressure is well known. Impulsively loaded vessels behave very differently. Generally the impulsive loading is induced by an internal explosion. This event can be unique (single shot) or repetitive. Design philosophy can be very different in both cases. If the loss of the vessel in a single detonation design can be accepted, it is absolutely not acceptable in a multiple shot design. Usually only elastic strains are induced in multiple shot vessels. This study focuses on vessels designed to withstand many impulsive loadings. Detonation vessels are used in many domains, such as demilitarization of munitions, explosive forming of metals (welding, cladding), manufacture of artificial diamonds, research on explosives and many more. In Belgium (amongst other countries), the technology of the controlled detonation chamber is used for the destruction of the remaining legacy of chemical munitions of the First World War. This detonation chamber has to withstand a large number of detonations. Knowledge of the structural response of the detonation chamber to different explosive charges is of high importance for both operational and safety reasons. In the operational use of the detonation vessels, a compromise must be made between inducing a small number of large impulses or a large number of small impulses on the vessel walls, with the vessel throughput/capacity as extra parameter. Recent investigations mainly focused on the vessel response to spherical charges [1-3]. Research on cylindrical explosive charges has been limited to open air effects $[4,5]$ or to a limited study of contained cylindrical charges [6]. The aim of this study is to provide a designer or operator with information about which charge parameter needs to be taken into account (or not) in order to assure a safe and rational use of the detonation vessel. This paper is focused on typical parameters of the explosive charge affecting the vessel response: the charge shape, the number of explosive charges, the charge location in the vessel and the location of initiation of the charge.

\section{Experimental setup}

\subsection{The vessel}

A 1/7 scale model of a $1000 \mathrm{~kg}$ detonation chamber, designed and manufactured by KOBELCO, Japan, has been used for testing. The chamber is a single wall cylindrical vessel with semi-elliptical ends. The total length is about $2 \mathrm{~m}$ with an inner diameter of $0.7 \mathrm{~m}$. The vessel has a particular design. One half of the cylindrical part of the chamber has been monolithically fabricated with a single 30-mm layer of SA-350, Type LF3 high-strength, low-alloy ferritic steel. This section will be referred to as the monobloc part. The other portion is of composite construction with a total thickness of $33.2 \mathrm{~mm}$, having an inner 6-mm layer of Type 316 stainless steel, a 3.2-mm-thick layer of carbon steel, and four outer 6mm layers of SA-724, Grade A high-strength, low-alloy steel. This section will be referred to as the multilayer part (see Fig. 1). This multilayer design was applied 
to stop possible cracks from growing through the entire wall thickness. The two sections are circumferentially welded at the vessel mid-section. This mid-section will be referred to as the beltline. Due to the use of different steels and a different total thickness, which both have an important influence on the vessel response, comparison between the monobloc and the multilayer technology has proved to be difficult. Separating the effects of thickness, material properties and design technology was not possible in this case.

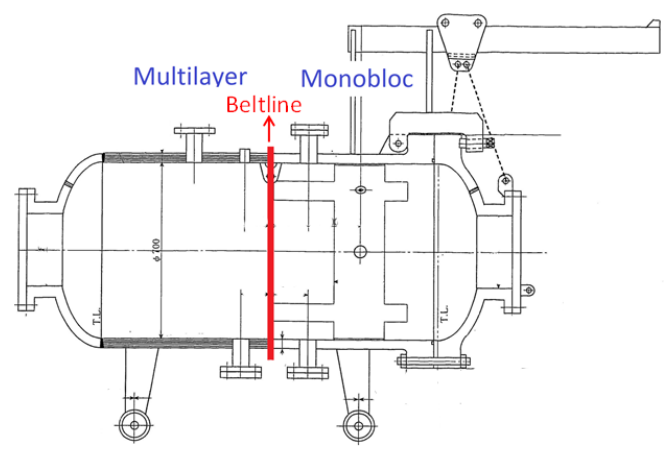

Figure 1: The scaled model detonation chamber and its particular design used for the experiments.

\subsection{The explosive charges}

The explosive used to induce an impulsive blast loading on the wall was a high explosive emulsion (blasting agent type, with main composition about $10 \%$ water, $6 \%$ oil, $80 \%$ ammonium nitrate, plus adequate emulsifier). The emulsion has been tested and a TNT-equivalent of 0.7 for impulse has been found. This means that $1 \mathrm{~kg}$ of emulsion produces the same impulse as $0.7 \mathrm{~kg}$ of TNT. This value has been confirmed for the very short range between the explosive charge and the vessel wall. This explosive emulsion can easily be fashioned into different shapes, which will influence the dynamic behavior of the detonation chamber [6]. Moreover, it is relatively safe to handle. Finally blast results from the detonation of emulsion charges showed an overall good reproducibility. Spherical and cylindrical charges with different length-to-diameter ratios have been tested. None of the charges discussed in this paper have led to any permanent deformation of the vessel wall.

\subsection{The measurement chain}

The vessel is equipped with high elongation strain gages. These gages and their cables are glued to the vessel to prevent failure because of the large accelerations of the vessel walls. The acquisition system consists of 48 channels, all able to acquire data at a rate of 50000 samples per second. A built-in low pass filter has been applied to reduce noise, and the measurements were remarkably reproducible. 
This proves the correct functioning of the measurement chain and the complete and reliable detonation of the emulsion explosive.

Gages are placed near the beltline, at $4 \mathrm{~cm}$ from the center of the welding line. These gages are assumed to be far enough from the welding line to avoid influence of that welding, and close enough to the vessel centerline to represent the central response adequately. Strain gages have also been placed on other locations of interest as well, for example near other welding lines and at the location of the explosive charges. Wherever possible, redundancy in the measurements gives extra confidence in the experimental data.

From the measured strain signals, peak values were determined. Over almost the entire cylindrical part, no strain growth has been observed. This means that the first peak is also the highest. Only at $5 \mathrm{~cm}$ of the semi-elliptical ends of the vessels, some strain growth has been observed. When fatigue damage is calculated, the major contribution would come from this first peak. Therefore, study of the influence of different charge parameters based on that first peak strain will lead to important recommendations for the use of multiple shot detonation vessels.

\section{Experimental results}

\subsection{Influence of the charge shape}

For this part of the study, explosive charges with two types of different shapes have been tested: spherical charges and cylindrical charges with different length-todiameter ratios (L/D). All these charges contained $2 \mathrm{~kg}$ of explosive emulsion. The dimensions of the used charges are given in Table 1. Figure 2 shows these charges inside the vessel. Smaller diameters have not been tested. The length would be at the limit to fit inside the vessel and the diameter could be too small to have good detonation (smaller than the critical diameter). Moreover, when filling such a charge, it is difficult to assure there are no (large) air gaps in the emulsion. Larger

Table 1: Different charge shapes with $2 \mathrm{~kg}$ emulsion used to study the influence of the charge shape.

\begin{tabular}{|c|c|c|c|}
\hline Shape & Length $(\mathbf{c m})$ & Diameter $(\mathbf{c m})$ & L/D \\
\hline \hline Cylindrical & 9.0 & 16 & 0.57 \\
\hline Cylindrical & 16.1 & 12 & 1.34 \\
\hline Cylindrical & 23.2 & 10 & 2.31 \\
\hline Cylindrical & 37.2 & 8 & 4.52 \\
\hline Cylindrical & 64.3 & 6 & 10.72 \\
\hline Cylindrical & 92.6 & 5 & 18.52 \\
\hline Spherical & - & 15.1 & - \\
\hline
\end{tabular}




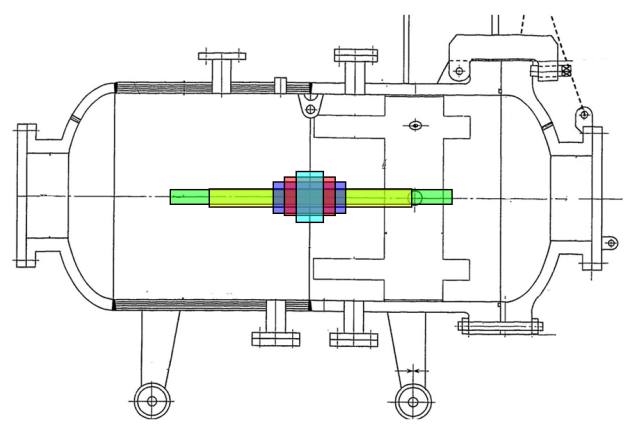

Figure 2: The different charges of $2 \mathrm{~kg}$ in the vessel, fired to study the influence of the shape of the charge.

diameters (and very short lengths) are practically difficult to manufacture as well, and do not really have any operational significance either.

The experimental peak strains on the beltline (which coincides with the charge centerline) are shown in Fig. 3. Lower peak strains have been measured in the multilayer section, because of its higher yield strength and larger total thickness. The highest peak strains were obtained for L/D about 2 to 3. Generally speaking, peak strains are higher for cylindrical charges than for the spherical charge.

It is obvious from these results that the shape of the explosive charge has a major influence on the peak deformation. Whenever possible, a spherical shape of the charge is preferable in order to have the smallest deformation of the vessel. This, in turn, will lead to the longest fatigue lifetime. However, a spherical charge concentrates the highest deformation at one location on the vessel wall. A longer cylindrical charge on the contrary has the benefit of spreading the loading over a larger zone. Figure 4 shows the distribution of the peak strains on the wall in function of the distance to the beltline. Peak strains on the beltline are lower for a

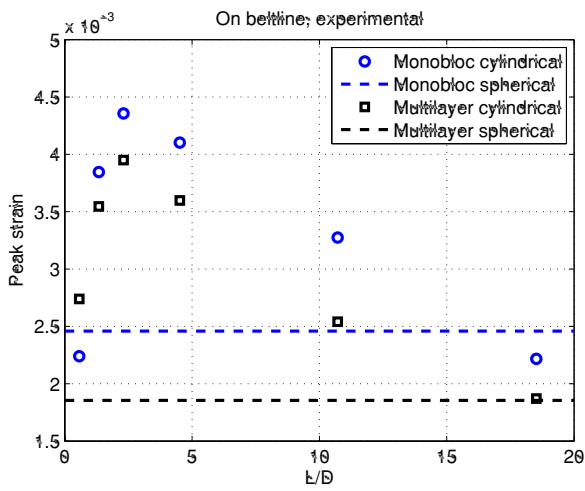

Figure 3: Experimental results on the beltline for different length-to-diameter ratios. 


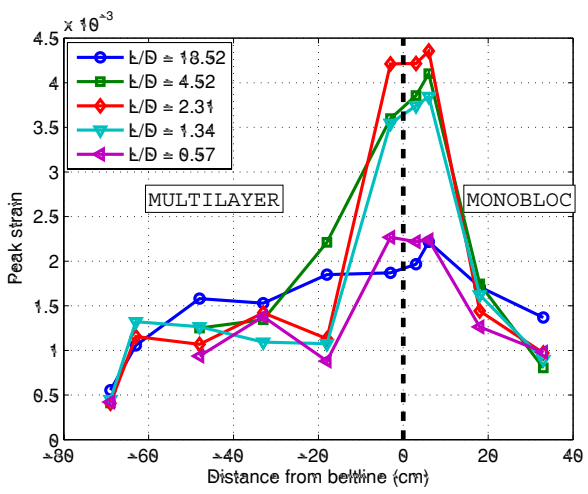

Figure 4: Peak strains on the vessel wall for different length-to-diameter ratios.

long, thin charge than for a short, thick charge. But some distance away from that beltline, the peak strains for the longer charge will be (slightly) higher. Instead of one single section of the cylinder being heavily loaded, a larger part is being moderately deformed. Whichever situation is preferable depends on the operator's choice. As it will be shown further, changing the location of the charge inside the vessel can be a solution when the charge effect is limited to a small section of the vessel wall.

All previous conclusions are also in good agreement with numerical simulations [7]. Numerical simulations with Ansys Autodyn ${ }^{\circledR}$ [8] showed that the influence of the charge shape depends on the distance from the charge. Simulations have been run with cylindrical charges with different length-to-diameter ratios and with spherical charges, all with an equal mass of $2 \mathrm{~kg}$. Reflected impulses (proportional to peak deformation) have been measured at various distances from the charge.

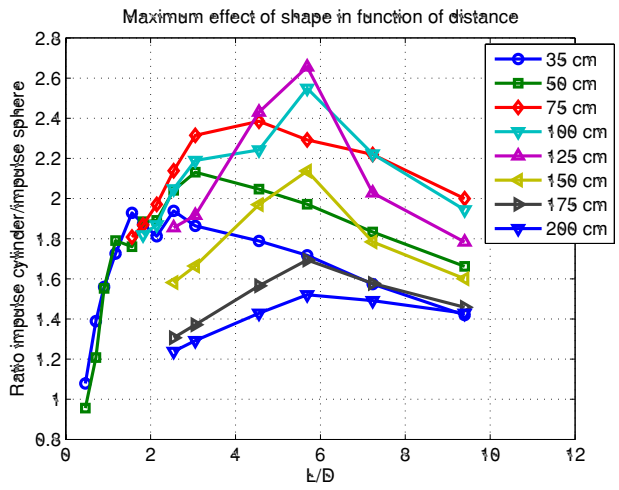

Figure 5: Effect of the shape of the charge (L/D) with respect to a spherical charge in function of the distance between the charge and the wall. 
For each distance and each cylindrical charge, the ratio between the impulse for the cylindrical charge and the spherical charge was calculated (Fig. 5). For each distance, ranging from 35 to $200 \mathrm{~cm}$, there is a particular L/D giving a maximal reflected impulse. That specific $\mathrm{L} / \mathrm{D}$ varies with increasing distance, from 1.5-2.5 at $35 \mathrm{~cm}$ to 5.5 at $1.25 \mathrm{~m}$ and more. That means that the charge leading to the highest deformation will depend on the diameter of each vessel.

\subsection{Double versus single charges}

In some applications, for example in the case of the destruction of some munitions, it is possible to use two half charges instead of one large charge. One single big package of munitions can be destroyed; or two smaller of those packages can be made and destroyed simultaneously in the vessel. The throughput of the system and the total amount of explosive will be identical, but one configuration causes smaller deformations to the vessel. This solution should be preferred, as it will lead to longer fatigue lifetimes.

In the small scale vessel, two types of shots have been fired to study the effect of split charges. The reference case was the cylindrical charge of $2 \mathrm{~kg}$ of emulsion with a length-to-diameter ratio of 1.34, placed centrally in the vessel (see Fig. 6, case 2)). This charge was then split into two cylindrical charges of $1 \mathrm{~kg}$ (with a length of $8 \mathrm{~cm}$ and a diameter of $12 \mathrm{~cm}$ ), separated by $60 \mathrm{~cm}$ (see Fig. 6, case 1). This distance was chosen because it places the $1 \mathrm{~kg}$ charges at the center of the monobloc and the multilayer section. It is obvious that a longer or shorter distance between the charges could lead to different conclusions. Both half charges were set up to be initiated simultaneously.

Figure 7(a) shows strain measurements near the beltline for both configurations. It is clearly visible that the peak strain for the single $2 \mathrm{~kg}$ charge is much higher than for the split charges. This is logical, as the $2 \mathrm{~kg}$ charge is located near the vessel beltline, and contains twice as much explosive. Figure 7(b) also shows

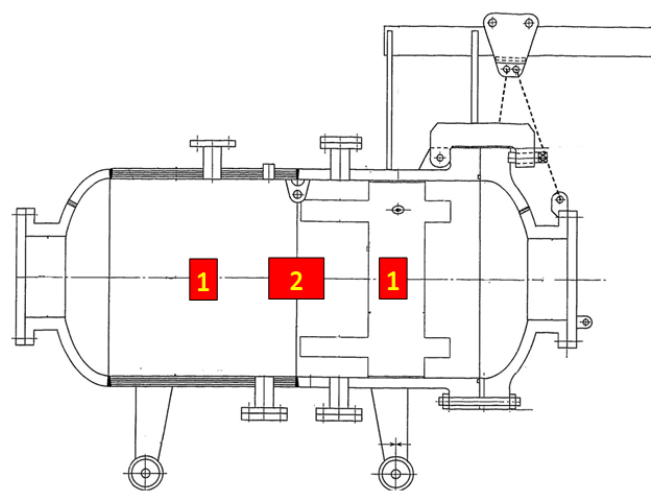

Figure 6: Position of the charges in the double charge tests. 1: positions of the two charges of $1 \mathrm{~kg}$. 2: position of the single $2 \mathrm{~kg}$ charge. 


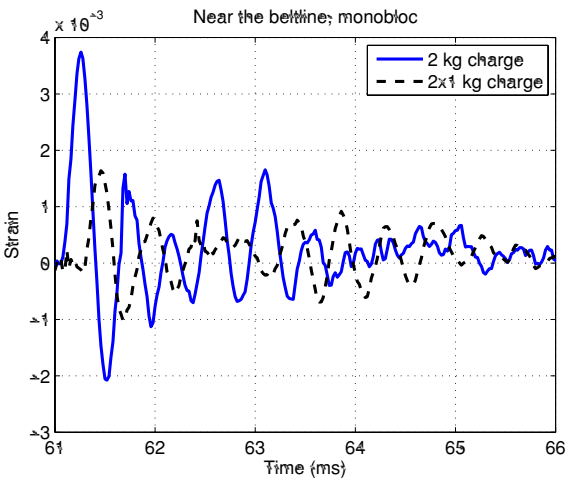

(a) Experimental strain near the beltline, monobloc section

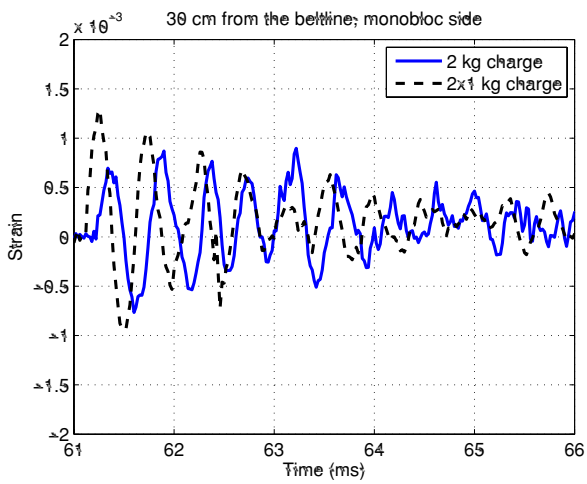

(b) Strain at the location of the $1 \mathrm{~kg}$ charge, monobloc section $(30 \mathrm{~cm}$ from the beltline)

Figure 7: Experimental results (measured strains) for one single $2 \mathrm{~kg}$ charge and two split (half) charges.

measured strains, but this time on the location where one of the $1 \mathrm{~kg}$ charges is situated (location 1 in Fig. 6). One notices that the peak strains for the shot with split charges and for the shot with a single $2 \mathrm{~kg}$ charge are similar at this location, which is the most heavily loaded by the $1 \mathrm{~kg}$ charge. Other measurements confirm that, when a charge is split into two equal parts, there is no location on the vessel where this leads to a larger response than in the case of a single charge. This conclusion is very important, as it indicates very clearly that the method of dividing a charge into two equal (half) parts leads to a significantly smaller vessel response. Whenever possible, this method should be applied by the vessel operator in order to extent the vessel lifetime.

Experiments have also shown that a small delay in initiation between the two half charges does not lead to significant differences in peak strain. There is no need for a particular way of initiating both charges simultaneously, and two standard detonators can be used for the easiest ignition of both charges. 


\subsection{Charge location in the vessel}

In some cases, when fragments are generated in the explosive event, changing the location of the explosive charge in the vessel is necessary to spread the damage of fragments over the entire vessel. The study with the double charges has allowed as well to determine the influence of the charge location in a cylindrical vessel. Generally, the cylindrical part of a vessel has some length where the explosive charge can be placed; the center is not the only possible location. Figure 8(a) shows a comparison between two strain measurements. The first signal was measured at the location of a $1 \mathrm{~kg}$ charge in a double charge shot (location A in Fig. 8(b)). The second signal is a strain measurement on the beltline weld for an identical $1 \mathrm{~kg}$ charge placed in the vessel center (location B in Fig. 8(b)). At the location of the charge, the initial response is the same, wherever the charge is located in the vessel (on the beltline or at some off-center position). Similar conclusions have been drawn on the multilayer section.

This is interesting when a vessel will be used with charges in varying locations. Moving the explosive charge in the vessel will not only be useful to spread the possible damage of fragments over the entire vessel, but also for spreading fatigue damage over a larger area.

\subsection{Influence of initiation}

For cylindrical explosive charges, it is well known that the location of initiation has an influence on the shock wave pattern around the charge [9]. As the impulse drives the vessel motion, the location of initiation is therefore of a potential influence. Experiments with two different initiation locations have been conducted, both with $2 \mathrm{~kg}$ charges placed centrally in the vessel. The explosive charge has been initiated at one end in most cases, and at the opposite end in one other case.

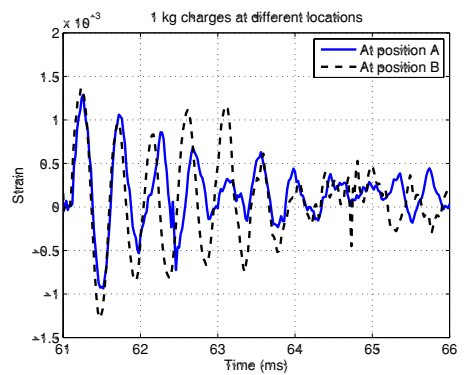

(a) Measured strains at the location of $1 \mathrm{~kg}$ charges in location $\mathrm{A}$ and $\mathrm{B}$

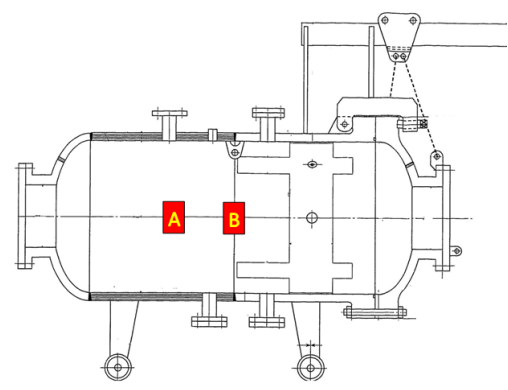

(b) Indications of positions of the $1 \mathrm{~kg}$ charge. Position A is off-center and position B is at the beltline

Figure 8: Comparison between the vessel responses for $1 \mathrm{~kg}$ charges at different locations. 


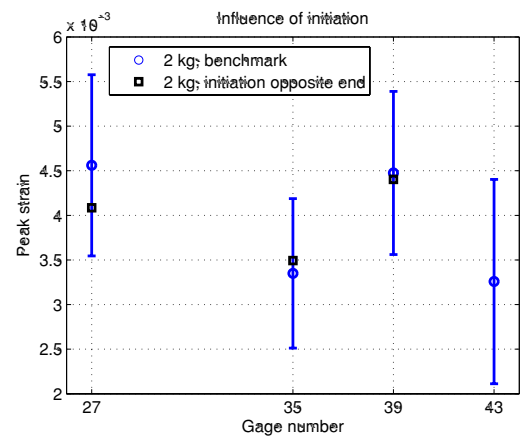

Figure 9: Peak strains for different gages near the vessel beltline with different initiation locations.

Figure 9 shows experimental peak strains for gages near the vessel beltline (also the charge centerline in this case). The figure shows the average peak strain with one standard deviation for six identical shots with a $2 \mathrm{~kg}$ standard cylindrical charge and initiation at one end. The strain measurements are generally well reproducible, but a small number of shots with larger explosive masses (3 to $4 \mathrm{~kg}$ ) during the test campaign have led to some hardening of the vessel material. This in turn leads to lower peak strains for the following $2 \mathrm{~kg}$ shots. This effect explains why the standard deviation is not as small as it could be expected. Figure 9 also shows the results for the peak strain of the shot with the initiation at the opposite end of the explosive charge. The peak strains are clearly nicely within the range of peak strains of the shots with standard initiation. As shown in [9], changing the location of initiation of a cylindrical charge does not (significantly) change the location and value of the peak impulse. The influence of the location of the initiation on the peak pressure is more important, but only impulse is the driving force in this case. This explains why modifying the initiation location does not change the vessel response.

This shows that the location of initiation is not of major influence on the vessel response. An operator can select the most convenient location of initiation without affecting the vessel response.

\section{Conclusion}

The response of impulsively loaded vessels to varying explosive charges has been studied. The most important factor has been shown to be the charge shape. Spherical charges are usually considered during the design phase of a detonation vessel. However, compared to these spherical charges, cylindrical charges with different length-to-diameter ratios can create significantly larger impulses. For each distance from the charge, one value of length-to-diameter ratio will lead to the most important vessel response. Knowledge of this particular shape is essential for the vessel operator. This study has shown as well that splitting a large charge 
into two equal half charges can reduce the vessel response, without reducing the chamber capacity. A rational use of the detonation vessel can significantly extend the vessel lifetime. Varying the location of the explosive charge in a cylindrical vessel is another possible method of spreading damage over the entire vessel wall instead of focusing it on one single location. This has been shown as another way to extend the vessel lifetime. Finally it has been shown that the location of initiation of the explosive charge is of little influence on the blast effect and the vessel response. Therefore an operator can simply choose the most practical location for initiation of the charge.

\section{Acknowledgements}

The authors thank Dr Nickell from Applied Science and Technology, San Diego, Texas for many fruitful discussions which have greatly helped this study to advance. The authors also express their gratitude to Dr Asahina from KOBELCO, Japan, for the intellectual, technical and logistic support during the experimental phase.

\section{References}

[1] Whenhui, Z., Honglu, X., Guangquan, Z. and Schleyer, G., Dynamic response of cylindrical explosive chambers to internal blast loading produced by a concentrated charge. Int J Impact Engng, pp. 831-845, 1997.

[2] Duffey, T., Rodriguez, E. and Romero, C., Design of pressure vessels for high strain rate loading: dynamic pressure and failure criteria, 2002. WRC Bulletin 477.

[3] Rodriguez, E., Nickell, R. and Pepin, J., Design Considerations for Blast Loads in Pressure Vessels. Transactions, Structural Mechanics in Reactor Technology 19, IASMiRT, 2007.

[4] Ismail, M. and Murray, S., Study of the Blast Waves from the Explosion of Nonspherical Charges. Propellants, Explosives and Pyrotechnics, 18, pp. 132-138, 1993.

[5] Held, M., Impulse Method for the Blast Contour of Cylindrical High Explosive Charges. Propellants, Explosives, Pyrotechnics, 24, pp. 17-26, 1999.

[6] Rushton, N., Schleyer, G.K., Clayton, A.M. and Thompson, S., Internal explosive loading of steel pipes. Thin-Walled Structures, pp. 870-877, 2008.

[7] Simoens, B., Lefebvre, M., Nickell, R., Asahina, J. and Minami, F., Analysis of the Dynamic Response of a Controlled Detonation Chamber. Journal of Pressure Vessel Technology, 133, 2011.

[8] Ansys, Autodyn ${ }^{\circledR}$, Explicit Software for Non-Linear Dynamics.

[9] Simoens, B., Lefebvre, M. and Minami, F., Influence of Different Parameters on the TNT-Equivalent of an Explosion. Central European Journal of Energetic Materials, 8(1), pp. 53-67, 2011. 